\title{
Aortic regurgitation and left ventricle remodeling on cardiac magnetic resonance and transthoracic echocardiography
}

\author{
Maciej Haberka', Mariusz Bałys', Zbigniew Gąsior', Bartłomiej Stasiów² \\ 'Department of Cardiology, School of Health Sciences, Medical University of Silesia, Katowice, Poland \\ ${ }^{2}$ Department of Electroradiology, School of Health Sciences, Medical University of Silesia, Katowice, Poland
}

\section{Editorial}

by Markl et al.

see p. 945

\section{Correspondence to: Prof. Maciej Haberka, $\mathrm{MD}, \mathrm{PhD}$, \\ Department of Cardiology, Medical University of Silesia Ziołowa 45/47, \\ 40-635 Katowice, Poland, phone: +48 322527407 e-mail: mhaberka@sum.edu.pl Copyright by the Author(s), 2021 \\ Kardiol Pol. 2021; 79 (9): 965-971; DOl: 10.33963/KP.a2021.0047 Received: April 6, 2021 Revision accepted: June 25, 2021 Published online: June 26, 2021}

\begin{abstract}
A B S T R A C T
Background: Transthoracic echocardiography (TTE) is the first imaging modality used to assess aortic regurgitation (AR). However, it is not possible to provide precise quantification in all patients.

Aim: Our aim was to compare TTE and cardiovascular magnetic resonance (CMR) measurements in grading AR and left ventricle (LV) remodeling.

Methods: A total of 51 consecutive patients with AR in TTE (New York Heart Association I/II, 55\%/38\%) were enrolled into the study and 49 individuals (age, 57.1 [14]; 61\% males) underwent a non-contrast CMR (2 patients excluded) obtained on 1.5 T system (GE Optima MR450w).

Results: The comprehensive quantitative grading with AR volume (AR vol) and regurgitant fraction (RF) were measurable in TTE in 24 cases and showed an association with CMR parameters (AR vol: $r=0.75$; $P<0.001$ and RF: $r=0.55 ; P<0.01)$. CMR revealed larger LV end-diastolic volumes (EDV) (185.5 [61] vs 158.4 [61] $\mathrm{ml} ; P=0.03$ ) and a trend towards higher left ventricular ejection fraction (59\% [8] vs 56\% [8]; $P=0.08)$. The association of AR vol and LV EDV was stronger in CMR $(r=0.85 ; P<0.0001)$ compared to $\operatorname{TTE}(r=0.6 ; P=0.001)$. The inter-modality agreement (TTE-CMR) in AR grading was low $(K=0.15)$, with highly concordant grading in mild AR (91\%).

Conclusions: CMR provides a comprehensive assessment of AR severity and LV remodeling with a weak or a moderate agreement with TTE.
\end{abstract}

Key words: aortic regurgitation, cardiovascular magnetic resonance, echocardiography

Kardiol Pol 2021; 79, 9: 965-971

\section{INTRODUCTION}

Transthoracic echocardiography (TTE) is the main imaging modality used to assess patients with chronic aortic regurgitation (AR). It is used for screening, grading, and monitoring patients with AR and mild symptoms. Current guidelines recommend aortic valve (AV) replacement or repair in patients with overt symptoms and/or severe left ventricle enlargement or systolic dysfunction [1]. The AR is usually well tolerated by most patients over several years and it is crucial to detect the optimal time to surgery and when the clinical prognosis is reduced. However, due to the limitations of ultrasound and the complex nature of AV disease, it is not possible to provide precise quantification of $A R$ and dimensions of the left ventricle (LV) in all patients. Therefore, our aim was to compare TTE and cardiovascular magnetic resonance (CMR) measurements in grading $A R$ and $L V$ remodeling in patients with chronic AR.

\section{METHODS}

\section{Study population}

All the consecutive patients scheduled for routine TTE in the echocardiography laboratory were screened (2018-2019) and 51 patients were included in the study group after consideration of exclusion criteria. The chronic regurgitation of the native aortic valve (with or without concomitant stenosis) was the inclusion criterion. 
WHAT'S NEW?

First, the study showed only a weak or moderate intermodality agreement between transthoracic echocardiography (TTE) and cardiovascular magnetic resonance (CMR) in quantitative aortic regurgitation (AR) grading. Second, the CMR-TTE compatibility was higher in patients with central jets with no associations with the degree of aortic valve (AV) degeneration or the Carpentier classification. Third, CMR provided significantly larger left ventricular end-diastolic volume (LV EDV) compared to TTE, which is especially important in patients with AR. Fourth, there was a significant association in LV EDV between the modalities, but CMR showed a stronger association between the quantitative AR parameters and LV EDV. The study supports better implementation of CMR into clinical practice, especially in patients with a moderate AR.

The main exclusion criteria were as follows: acute aortic regurgitation and/or endocarditis of any native valve, aortic valve prosthesis, any stenosis or more than mild mitral regurgitation, acute coronary syndrome or decompensated congestive heart failure in prior 1 month, infectious diseases in prior 1 month, significant anemia, and contraindications to $C M R$.

This was a prospective single-center study performed in accordance with the principles of the Declaration of Helsinki and the Bioethics Committee of the Medical University of Silesia. All patients signed the informed consent. This work was supported by the research non-commercial grant from the Medical University of Silesia (KNW-1027/K/9/K).

\section{Echocardiography}

Echocardiography (TTE) was performed with a commercially available 2-dimensional imaging system (General Electric company Vivid e9, Milwaukee, WI, USA). All the patients were scanned in the left lateral decubitus position and each of the examinations followed the EACVI/ASE 2015 recommendations for cardiac chamber quantification by echocardiography in adults [2]. Left ventricular ejection fraction (LVEF) was calculated with the biplane Simpson method (without an ultrasound contrast) and LV mass was estimated using the linear method and Cube formula [2]. Given the main aim of the paper, the grading of the AR was based on the extensive multiparametric approach following the 2017 ASE recommendations for noninvasive evaluation of native valvular regurgitation [3]. The integrative AR measurements were obtained in all patients using qualitative, semi-quantitative (SQ) and quantitative (Q) parameters depending on the quality of the acoustic window and the type of AR itself. Each of the TTE followed the steps of the $S Q$ and $Q$ described in the guidelines [3]. The AR volume (AR vol) and AR regurgitant fraction (AR RF) were estimated using the Doppler method based on proximal isovelocity surface area (PISA). The final severity of $A R$ was a multiparametric assessment with the focus on Q parameters or SQ, if PISA method was not measurable (acoustic window, asymmetric jet, multiple jets). The following quantitative criteria for the AR severity were used: severe (AR vol $\geq 60 \mathrm{ml} ; \mathrm{RF} \geq 50 \%$; $E R O \geq 0.3 \mathrm{~cm}^{2}$ ), moderate $\left(A R\right.$ vol $\left.=30-59 \mathrm{ml} ; \mathrm{RF}=30 \%-49 \% ; E R O=0.1-0.29 \mathrm{~cm}^{2}\right)$ and mild (AR vol $<30 \mathrm{ml}$; $\mathrm{RF}<30 \%$; $\mathrm{ERO}<0.1 \mathrm{~cm}^{2}$ ) [3]. The degree of $A V$ calcifications was based on the subjective assessment, where " 0 " was for elastic leaflets with no calcifications and "3" was for hyperechogenic severe calcifications. The angle of AR was assessed manually by the single observer as the angle between the axis of the aortic root and the axis of the jet in the parasternal long-axis view. The AR jet angle 0 was central and the more asymmetric jet (in either of the sides) the larger the AR angle.

All the TTE images were obtained, stored anonymously, and then analyzed offline by a single observer (MB) blinded to patient clinical characteristics and CMR results.

\section{Cardiovascular magnetic resonance imaging}

The CMR images were acquired on 1.5T system (GE Optima MR450w, GE Healthcare, Wisconsin, WI, USA) with a dedicated phased-array cardiac coil. The study CMR protocol included a non-contrast examination with a multi-planar cine steady-state free precession (SSFP) acquisitions and flow visualization using phase-contrast (PC) flow imaging. Cardiac chambers volumes and functions were analyzed by SSFP in several planes, including 2-chamber, 4-chamber, orthogonal LV outflow track, and parallel short-axis planes covering both atria and ventricles. The typical scan parameters used were TE/TR (time to echo/time of repetition) 1.9/4.3 ms, slice thickness 4-8 mm (no interslice gap), and temporal resolution $30-40 \mathrm{~ms}$. The SSFP planes for the aortic valve complex and ascending aorta were placed perpendicular to the aortic root. The through-plane PC flow imaging was obtained at the slices perpendicular to the axis of flow with the positions just above the valve and velocity encoding maximum values set at $200 \mathrm{~cm} / \mathrm{s}$. The PC imaging was repeated, and the position of the slice and maximum velocity values were modified to avoid aliasing or artifacts [4]. The severity of AR was based on AR vol and AR RF obtained in PC imaging and volumetric data calculated from cine images and manual endocardial tracings. The CMR grades of AR were defined according to the 2017 ASE guidelines and the criteria for AR vol and RF were similar as described above for echocardiography [3].

\section{Statistical analysis}

The results presented in the manuscript are expressed as means (standard deviation [SD]) for normally distributed 
variables or medians (interquartile range [IQR]) for abnormal distribution. In the case of descriptive data, a number (percentage) was used. The distribution was tested for normality with the Kolmogorov-Smirnov test. Baseline clinical parameters and the measures were compared between the subgroups using the t-tests for the normally distributed continuous variable (Student's t-test); in case of abnormal distribution, the Mann-Whitney $U$ test was used. Associations between parameters were assessed using Pearson correlation analysis (parametric variables). The Bland-Altman analysis was performed to measure the agreement between the main parameters of CMR and TTE (AR vol and AR RF). Intermodality agreement for AR severity classification was performed using $\mathrm{k}$ statistics. The calculated kappa coefficients were graded as follows: 0-0.2 low, 0.2-0.4 fair, 0.4-0.6 moderate, $0.6-0.8$ good, and $>0.8$ excellent [5]. A value $P<0.05$ was considered statistically significant. Statistical analysis was undertaken using Medcalc software (version 19.1, Osten, Belgium).

Table 1. Clinical characteristics of the study group

\begin{tabular}{|c|c|}
\hline Age, years, mean (SD) & $57.1(14.2)$ \\
\hline Female/male, n (\%) & $19(39) / 30(61)$ \\
\hline Diabetes, n (\%) & $6(12)$ \\
\hline Dyslipidemia, n (\%) & $45(91)$ \\
\hline Hypertension, n (\%) & $41(83)$ \\
\hline Smoker or ex-smoker, n (\%) & $7(14)$ \\
\hline Ischemic heart disease, $\mathrm{n}(\%)$ & $22(45)$ \\
\hline Prior Ml, n (\%) & $0(0)$ \\
\hline Body mass index, $\mathrm{kg} / \mathrm{m}^{2}$, mean (SD) & $26.4(3.6)$ \\
\hline Overweight/obesity, n (\%) & $24(48) / 8(16)$ \\
\hline Body surface area, $\mathrm{m}^{2 \mathrm{a}}$, mean (SD) & $1.93(0.18)$ \\
\hline \multicolumn{2}{|l|}{ NYHA class, n (\%) } \\
\hline 1 & $27(55)$ \\
\hline$\|$ & $19(38)$ \\
\hline III & $3(7)$ \\
\hline IV & 0 \\
\hline \multicolumn{2}{|l|}{ CCS class, n (\%) } \\
\hline 0 & 39 (79.6) \\
\hline 1 & $1(2)$ \\
\hline ॥ & $6(12.2)$ \\
\hline III & $3(6.2)$ \\
\hline IV & 0 \\
\hline \multicolumn{2}{|l|}{ Aortic valve disease, $\mathrm{n}(\%)$} \\
\hline Bicuspid aortic valve & $14(28.5)$ \\
\hline Aortic valve stenosis & $20(40)$ \\
\hline \multicolumn{2}{|l|}{ Aortic valve calcifications, n (\%) } \\
\hline 0 & $14(28)$ \\
\hline 1 & $16(32)$ \\
\hline 2 & $15(30)$ \\
\hline 3 & $4(10)$ \\
\hline \multicolumn{2}{|l|}{ Aortic valve regurgitation, $\mathrm{n}(\%)$} \\
\hline Multiple jets in echocardiography & $7(14)$ \\
\hline Central/eccentric jet & $26(53) / 23(47)$ \\
\hline \multicolumn{2}{|l|}{ Jet angle, n (\%) } \\
\hline $0-30$ & $23(47)$ \\
\hline $31-60$ & $16(32)$ \\
\hline $61-90$ & $10(20)$ \\
\hline
\end{tabular}

Abbreviations: Ml, myocardial infarction; NYHA, New York Heart Association

\section{RESULTS}

\section{Clinical characteristics}

A total of 51 consecutive patients with AR in TTE were enrolled into the study and finally 49 individuals (age: 57.1 [14]; 61\% males) completed a non-contrast CMR (2 excluded for CMR contraindications).

The clinical characteristics, symptoms, and baseline parameters of AV disease are presented in Table 1. In brief, the study patients revealed cardiovascular risk factors, including dyslipidemia (91\%), hypertension (83\%), obesity $(16 \%)$, or diabetes (12\%). Ischemic heart disease was found in almost half of the patients (45\%), and most subjects showed moderate symptoms (New York Heart Association I or II).

Most individuals had some degree of AV calcification (72\%), which resulted in a mild (19 patients; $38 \%$ ) or a moderate (1 patient; $2 \%$ ) AV stenosis. Half of the subjects showed AR with eccentric jets, and multiple jets were found in $14 \%$ of cases.

\section{Left ventricle remodeling}

Left ventricle dimensions and systolic function were assessed in both modalities. The LV end-diastolic diameters (EDD) and end-diastolic volumes (EDV) measured in CMR were larger compared to TTE: 57.4 (7) $\mathrm{mm}$ vs 54 (6) $\mathrm{mm}$ $(P=0.02)$ and $185.5(61) \mathrm{ml}$ vs $158.5(61) \mathrm{ml}(P=0.03)$. However, there were no differences in the LV end-systolic diameters (35.6 [8] $\mathrm{mm}$ vs 35.1 [6] $\mathrm{mm} ; P=0.7$ ), end-systolic volumes (ESV) (78.5 [36] $\mathrm{ml}$ vs 71 [33.2] $\mathrm{ml} ; P=0.3$ ) and there was a trend towards higher LV ejection fractions in CMR compared to TTE (59 [8] \% vs 56.2 [8] \%; $P=0.08$ ). Despite some differences, there were significant associations between both modalities in LV EDV and ESV (Figures 1, 2), but not in LV EF. Moreover, LV mass calculated in TTE was overestimated compared to CMR (245 [84] g vs 152 [52] g; $P<0.0001)$, but there was also an association between modalities $(r=0.8 ; P<0.0001)$.

\section{Aortic regurgitation}

The main SQ measures found in TTE were as follows: jet/LVOT ranged from $15 \%$ to $65 \%$ (31\% [22-36]) and PHT was between $35 \mathrm{~ms}$ and $752 \mathrm{~ms}$ (404 ms [320-510]). The comprehensive quantitative assessment by the Doppler PISA method with AR vol and AR RF were measurable in TTE in 24 cases: AR vol $38 \mathrm{ml}$ (26-57 ml) and AR RF 43\% (33\%-57\%).

The CMR AR vol ranged from $5 \mathrm{ml}$ to $92 \mathrm{ml}$ (18 $\mathrm{ml}$ [6-39]) and AR RF ranged from $5 \%$ to $75 \%$ (21\% [7-36]). In the subgroup of 24 cases, the TTE AR vol was larger compared to CMR AR: $38.5 \mathrm{ml}(26-57)$ vs $18 \mathrm{ml}(6-39)(P<0.01)$ and TTE AR RF was higher compared to CMR RF: 43\% (33-57) vs $21 \%(7-36)(P<0.0001)$.

Both modalities showed significant associations in AR vol and AR RF (Figures 3, 4).

Both TTE and CMR provided the conclusions for AR grading, which were compared in all patients. The intermo- 


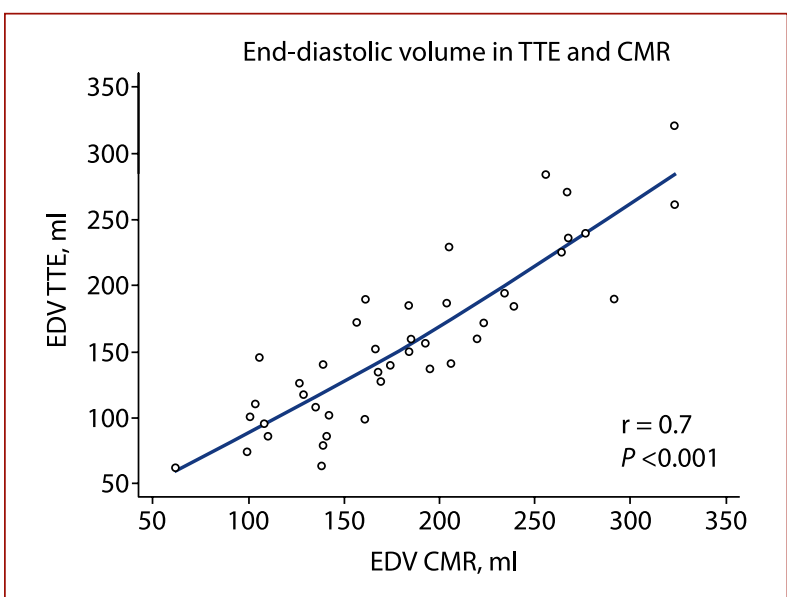

Figure 1. End-diastolic volume (EDV) on transthoracic echocardiography (TTE) and cardiovascular magnetic resonance (CMR). Pearson correlation

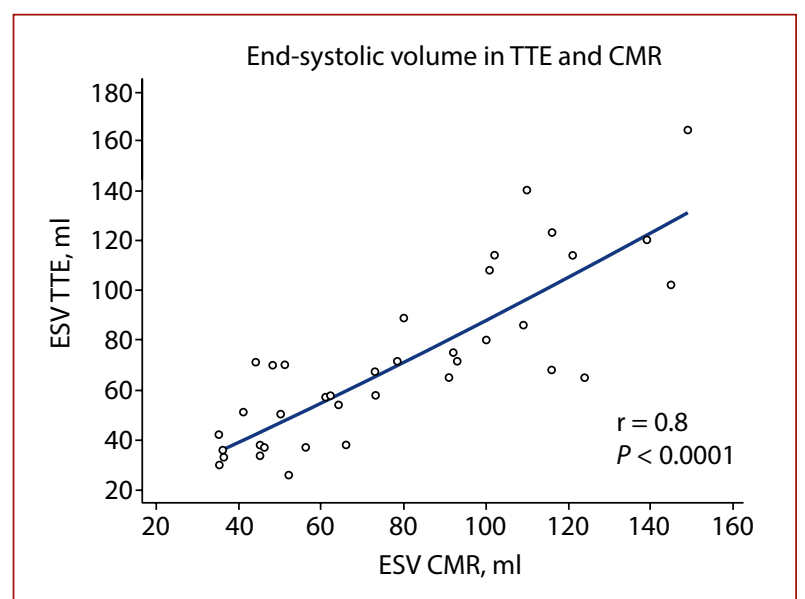

Figure 2. End-systolic volume (ESV) on transthoracic echocardiography (TTE) and cardiovascular magnetic resonance (CMR). Pearson correlation

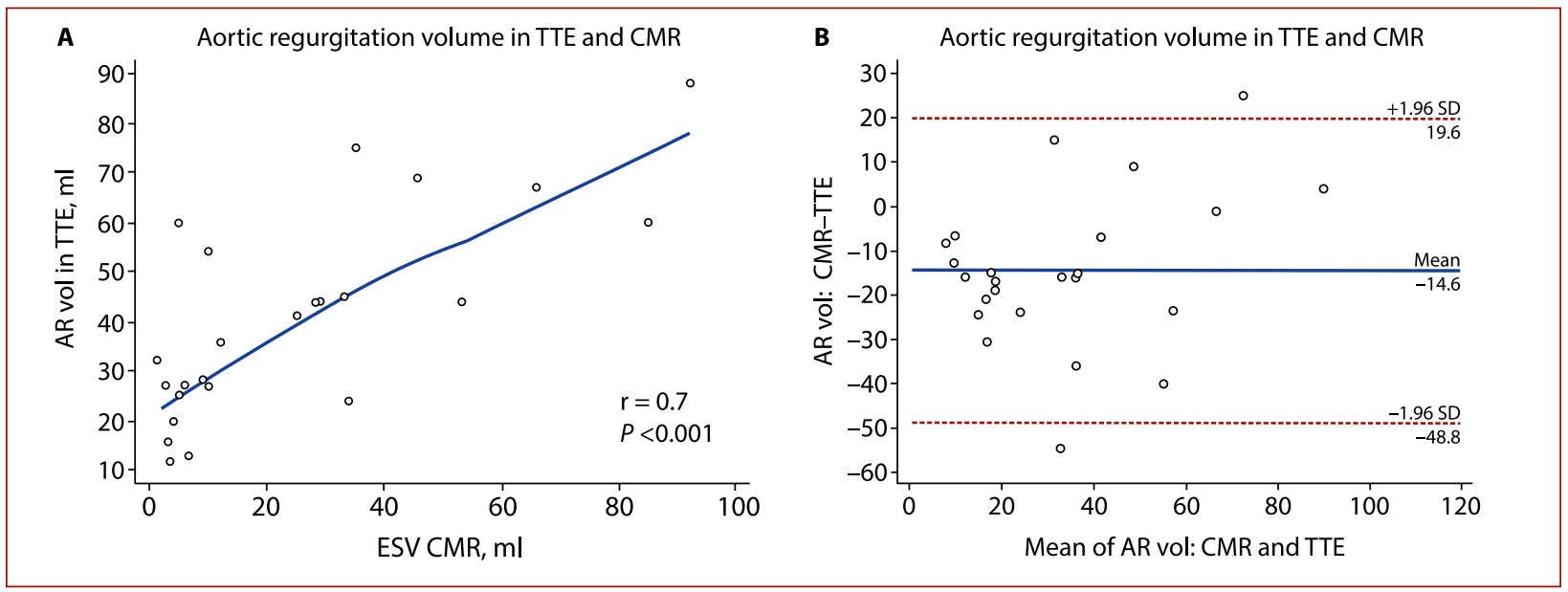

Figure 3. Aortic regurgitation volume (AR vol) on transthoracic echocardiography (TTE) and cardiovascular magnetic resonance (CMR) - Pearson correlation (A) and Bland-Altman plot (B)
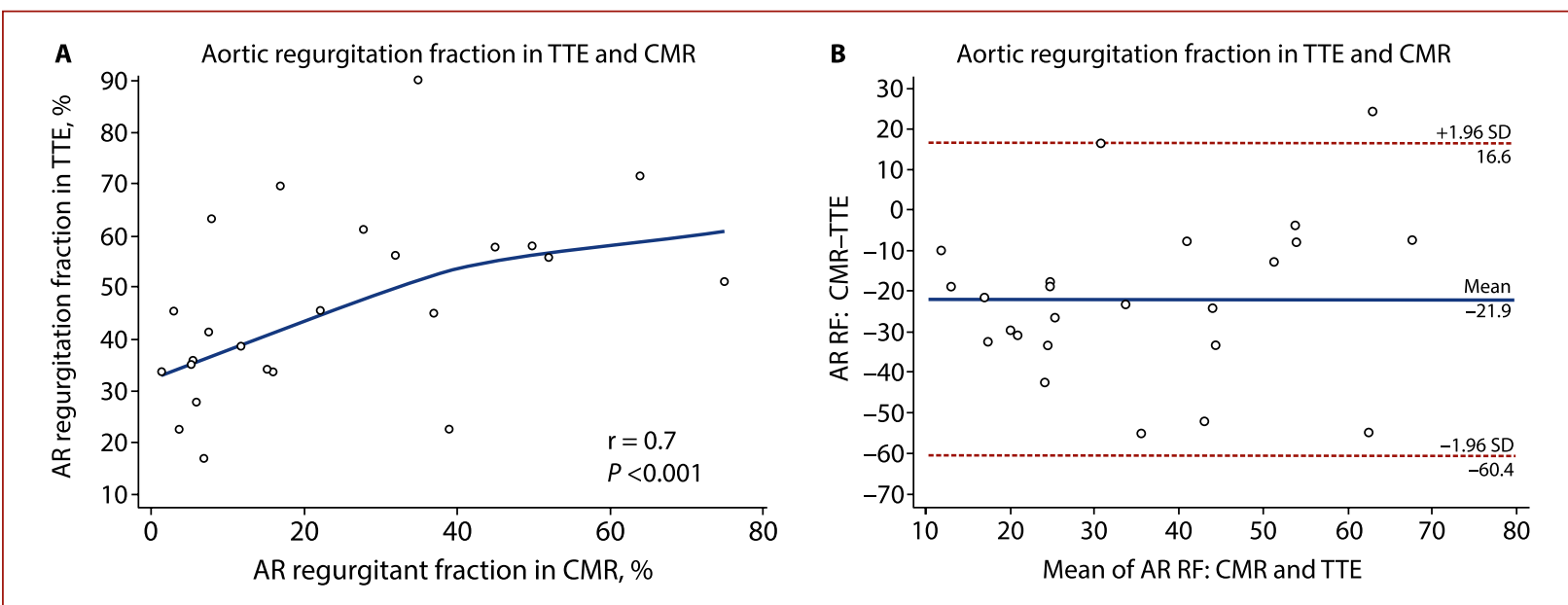

Figure 4. Aortic regurgitation - regurgitation fraction (AR RF) on transthoracic echocardiography (TTE) and cardiovascular magnetic resonance (CMR) — Pearson correlation (A) and Bland-Altman plot (B) 
Table 2. Aortic regurgitation grading in transthoracic echocardiography and cardiovascular magnetic resonance

\begin{tabular}{lccc} 
Transthoracic echocardiography & Mild & Moderate & Severe \\
Mild & 9 & 0 & 0 \\
Moderate & 22 & 9 & 4 \\
Severe & 1 & 1 & 3 \\
\hline
\end{tabular}

dality agreement (TTE-CMR) in AR grading across a mild, moderate and severe AR was low $(K=0.15, \mathrm{SE}=0.08 ; 95 \%$ $\mathrm{Cl},-0.006-0.309$ ) (Table 2). Both modalities provided similar conclusions mostly in mild AR (91\%). TTE overestimated the grade of AR in $51 \%$ and underestimated the AR severity in $15 \%$ of patients (Figure 5 ).

The rate of concordant AR severity between both modalities was significantly higher in 32 patients with jet angle $<40^{\circ}$ compared to 17 subjects with asymmetric jet angle $\geq 40^{\circ}$ (50\% vs $35 \%$; $P<0.05$ ). However, the degree of AV calcification or the mechanism of AR according to the Carpentier classification were not associated with the rate of concordant AR severity ( $P=\mathrm{ns})$.

The association of AR vol and LV EDV was stronger in CMR ( $r=0.85 ; P<0.0001)$ compared to TTE ( $r=0.6$; $P=0.001)$.

Myerson et al. showed that CMR AR RF $>33 \%$ or AR vol $>42 \mathrm{ml}$ have a high predictive value to identify patients who will develop symptoms and strong indication for surgery in the following years [4]. In our study group, 18 patients revealed at least one of the above parameters identifying those with worse prognoses. In this subgroup, TTE indi- cated a significant AR in 50\% (severe or moderate-to-severe) and underestimated AR in another $50 \%$ of subjects (mild-to-moderate in $45 \%$ and even mild in 5\%).

\section{DISCUSSION}

Our prospective study evaluated the incremental value of CMR over TTE for the assessment of patients with chronic AR. First, there was a moderate intermodality agreement between TTE and CMR in quantitative AR grading. Second, the CMR-TTE compatibility was higher in patients with central jets with no associations with the degree of AV degeneration or the Carpentier classification. Third, CMR provided significantly larger LV EDV compared to TTE, which is especially important in patients with AR. Fourth, there was a significant association in LV EDV between the modalities, but CMR showed a stronger association between the quantitative AR parameters and LV EDV.

Our results are based on the consecutive patients scheduled for the echocardiography lab and they are representative for clinical practice. There were patients with all the degrees of native AR, different AR mechanisms, and various severity of AV calcifications, including any grade of stenosis in $40 \%$ of cases. We found that the comprehensive quantitative grading by the Doppler PISA method was available in TTE only in half of the cases. While the PISA method is a major tool for mitral valve regurgitation [6], it is not well visualized in AR, except for significant regurgitations with preferably central jets, when a continuous wave Doppler may be used. Pirat et al. showed that regurgitant volumes in the 2D PISA method had a moderate correlation

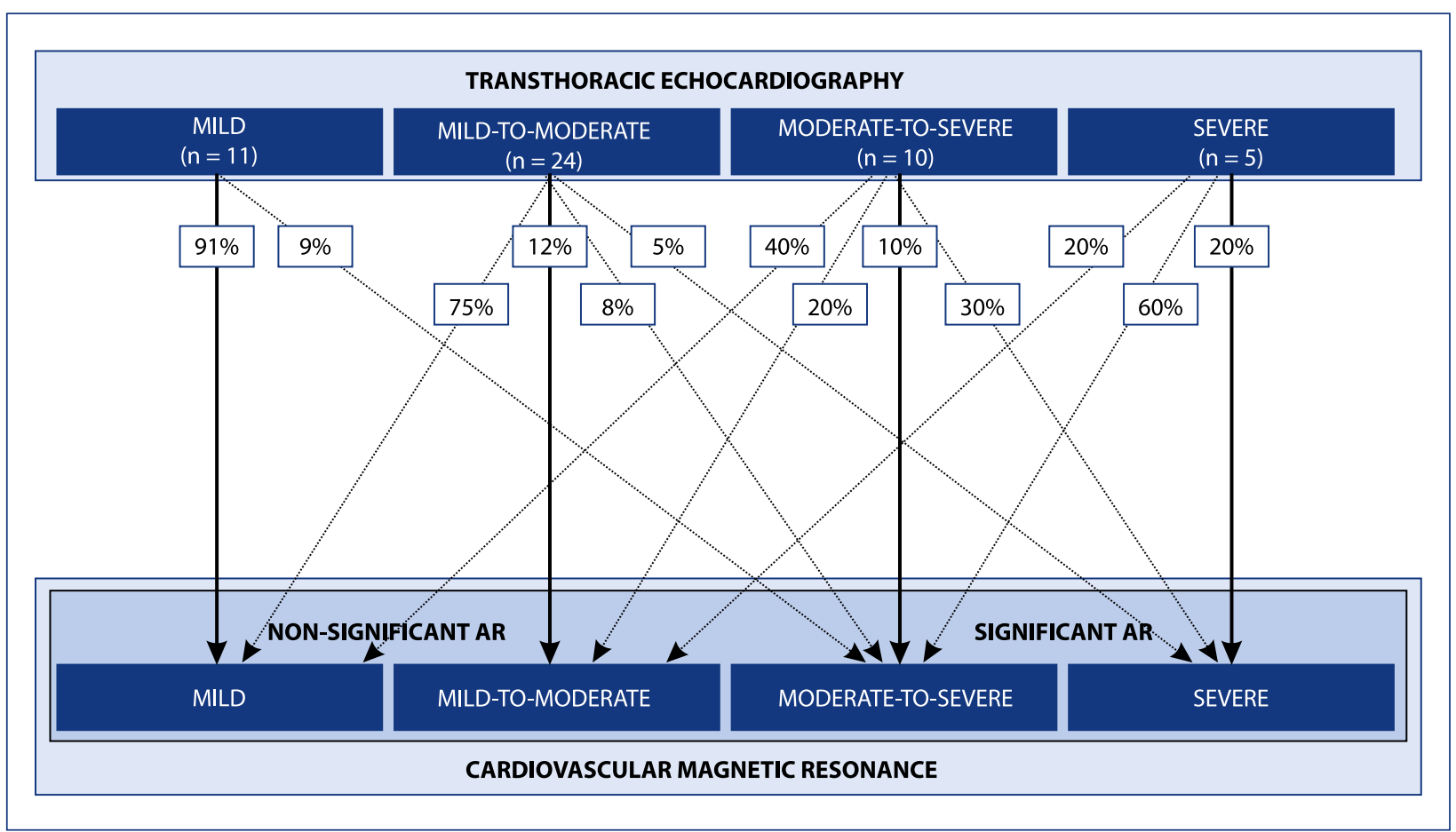

Figure 5. Flow chart - the primary grading of aortic regurgitation on transthoracic echocardiography (number of patients in brackets) and the final grading on cardiovascular magnetic resonance with the rates of patients (\%)

Abbreviations: AR, aortic regurgitation 
with the actual volumes in an in-vitro flow model for AR, with an accuracy dependent on the shape of the orifice [7]. The 3D PISA method would improve the accuracy of $A R$ quantification, but it requires high-quality images available in transesophageal echocardiography. The other TTE method for quantification (the SV method) is prone to variability mainly due to a 3-dimensional complex and dynamic shape of the mitral annulus and LV outflow tract. The minor change in echocardiographic view is subject to a major change in diameters and estimated volumes and it has higher variability in measurements [4].

Our study showed that the AR vol and AR RF were significantly larger in TTE compared to CMR. Given that TTE showed systematically lower volumes of LV, it is expected that AR RF would be systematically overestimated compared to CMR. Moreover, AR RF obtained in TTE showed only a moderate association with a reference $C M R$, which suggests that it should not be used in clinical practice. The conclusions of both modalities obtained in all study groups were similar in patients with mild AR. The concordance among the other three grades of AR was moderate or low and, in general, TTE overestimated most cases. However, we had only 5 patients with a severe AR in TTE. Cawley et al. compared both modalities and they also found that TTE used in 31 patients (SV method) overestimated AR vol with no differences in AR RF [8]. There was also only a moderate association between modalities in AR volumes. Gelfand et al. showed a moderate agreement in final AR grading in a small group of patients with AR [9]. Our study is one of the largest among few papers comparing CMR with echocardiography in patients with a native AR [10].

Previous reports showed that the CMR AR grading and $A R$ volumes revealed better predictive values for AV replacement (AVR) or heart failure and it showed a better correlation with an LV remodeling following AVR compared to echocardiography in a relatively small study groups $[11,12]$. Given the cut-offs for AR vol and AR RF in CMR provided by Myerson et al. [4], we found that TTE would underestimate the clinical prognosis in half of our study group in a relation to the same cut-off values.

We have used the same cut-off values for quantitative parameters (AR vol and AR RF) in both TTE and CMR, which were recommended in the latest guidelines [3]. There are various cut-offs suggested for AR grading in CMR [13, 14]. The evidence on the appropriate values for CMR is still very scarce and we are awaiting the specific guidelines. We used the CMR PC as the primary method for AR grading as it was shown to have lower variability compared to the CMR RVLV volume method [6]. Moreover, it was shown that this direct method of AR quantification has high accuracy and reproducibility [15-17].

LV remodeling and systolic function are the other key parameters for appropriate timing of cardiac surgery [1]. CMR is well evidenced to provide very accurate measurements of LV volumes, function, and mass [18, 19]. We found that CMR revealed significantly larger LV EDV compared to
TTE. It is in line with previous studies $[8,10]$. All our study patients had preserved LV systolic function and the relative differences were small. Therefore, our number of study patients might not have been large enough to provide statistical significance. Although there was an association between LV mass calculated in CMR and estimated in TTE, the linear method used in TTE led to a significant overestimation as it was found in previous reports [8]. Finally, our study showed that there was a stronger association between the quantification of AR and LV EDV in CMR compared to TTE, which is a similar result to the study by Uretsky et al. [20]. Aortic valve-sparing surgery is a recently evolving novel surgical therapeutic option with promising early effects and long-term clinical outcomes [21, 22]. However, it is a highly complex procedure requiring comprehensive imaging diagnostics and an experienced surgical team. Thus, CMR seems to be the best imaging modality for patients scheduled for AV repair.

\section{Study limitations}

Our study group included only 5 individuals with a severe AR, which limits the strength of our conclusions. We do not have a clinical follow-up yet and we cannot relate our results to clinical prognosis. All the valve regurgitations have a physiological variability in loading conditions dependent on blood pressure or heart rate, which could affect the measurements. However, it affects both modalities and our study group included only chronic AR. We did not use 3-dimensional measurements of LV volumes, which would improve the compatibility in measurements between TTE and CMR. However, 3D TTE is measurable only in patients with a good acoustic window.

\section{CONCLUSIONS}

In our study, CMR provided a comprehensive assessment of $A R$ severity and $L V$ remodeling with a moderate agreement with TTE and a better clinical predictive value. In clinical practice, echocardiography is the most reliable in patients with mild or non-significant AR and central jets with good conditions for a PISA method quantification. However, a comprehensive quantitative assessment by the PISA method was measurable in less than half of the study patients. There is a clear underestimation of the degree of LV remodeling in TTE and systematical difference in quantitative parameters of $A R$ between CMR and TTE. It suggests the need for new cut-off values in AR in both modalities. Our study supports also better implementation of CMR into clinical practice, especially in patients with a moderate AR.

\section{Article information}

Conflict of interest: None declared.

Funding statement: This work was supported by the research non-commercial grant from Medical University of Silesia (KNW-1$-027 / K / 9 / K)$

Open access: This article is available in open access under Creative Common Attribution-Non-Commercial-No Derivatives 4.0 International (CC BY-NC-ND 4.0) license, allowing to download articles and 
share them with others as long as they credit the authors and the publisher, but without permission to change them in any way or use them commercially. For commercial use, please contact the journal office at kardiologiapolska@ptkardio.pl.

How to cite: Haberka M, Bałys M, Gąsior Z, Stasiów B. Aortic regurgitation and left ventricle remodeling in cardiac magnetic resonance and transthoracic echocardiography. Kardiol Pol. 2021; 79(9): 965-971, doi: 10.33963/KP.a2021.0047.

\section{REFERENCES}

1. Baumgartner H, Falk V, Bax JJ. 2017 ESC/EACTS Guidelines for the management of valvular heart disease. Eur Heart J. 2017; 38(36): 2739-2791, doi: 10.1093/eurheartj/ehx391, indexed in Pubmed: 28886619.

2. Lang RM, Badano LP, Mor-Avi V, et al. Recommendations for cardiac chamber quantification by echocardiography in adults: an update from the American Society of Echocardiography and the European Association of Cardiovascular Imaging. J Am Soc Echocardiogr. 2015; 28(1): 1-39.e14 doi: 10.1016/j.echo.2014.10.003, indexed in Pubmed: 25559473.

3. Zoghbi WA, Adams D, Bonow RO, et al. Recommendations for noninvasive evaluation of native valvular regurgitation - a report from the American Society of Echocardiography developed in collaboration with the Society for Cardiovascular Magnetic Resonance. J Am Soc Echocardiogr. 2017; 30(4): 303-371, doi: 10.1016/j.echo.2017.01.007, indexed in Pubmed: 28314623.

4. Myerson SG, d'Arcy J, Mohiaddin R, et al. Aortic regurgitation quantification using cardiovascular magnetic resonance: association with clinical outcome. Circulation. 2012; 126(12): 1452-1460, doi: 10.1161/CIRCULATIONAHA.111.083600, indexed in Pubmed: 22879371.

5. Medvedofsky D, León Jiménez J, Addetia K, et al. Multi-parametric quantification of tricuspid regurgitation using cardiovascular magnetic resonance: A comparison to echocardiography. Eur J Radiol. 2017; 86 213-220, doi: 10.1016/j.ejrad.2016.11.025, indexed in Pubmed: 28027750.

6. Sköldborg V, Madsen PL, Dalsgaard M, et al. Quantification of mitral valve regurgitation by $2 \mathrm{D}$ and $3 \mathrm{D}$ echocardiography compared with cardiac magnetic resonance a systematic review and meta-analysis. Int J Cardiovasc Imaging. 2020; 36(2): 279-289, doi: 10.1007/s10554-019-01713-7, indexed in Pubmed: 31664679.

7. Pirat B, Little SH, Igo SR, et al. Direct measurement of proximal isovelocity surface area by real-time three-dimensional color Doppler for quantitation of aortic regurgitant volume: an in vitro validation. J Am Soc Echocardiogr. 2009; 22(3): 306-313, doi: 10.1016/j.echo.2008.11.031, indexed in Pubmed: 19168322.

8. Cawley PJ, Hamilton-Craig C, Owens DS, et al. Prospective comparison of valve regurgitation quantitation by cardiac magnetic resonance imaging and transthoracic echocardiography. Circ Cardiovasc Imaging. 2013; 6(1): 48-57, doi: 10.1161/CIRCIMAGING.112.975623, indexed in Pubmed: 23212272.

9. Gelfand EV, Hughes $\mathrm{S}$, Hauser $\mathrm{TH}$, et al. Severity of mitral and aortic regurgitation as assessed by cardiovascular magnetic resonance: optimizing correlation with Doppler echocardiography. J Cardiovasc Magn Reson. 2006; 8(3): 503-507, doi: 10.1080/10976640600604856, indexed in Pubmed: 16755839.

10. Lee JC, Branch KR, Hamilton-Craig C, et al. Evaluation of aortic regurgitation with cardiac magnetic resonance imaging: a systematic review.
Heart. 2018; 104(2): 103-110, doi: 10.1136/heartjnl-2016-310819, indexed in Pubmed: 28822982

11. Neisius U, Tsao CW, Hauser TH, et al. Aortic regurgitation assessment by cardiovascular magnetic resonance imaging and transthoracic echocardiography: intermodality disagreement impacting on prediction of post-surgical left ventricular remodeling. Int J Cardiovasc Imaging. 2020; 36(1): 91-100, doi: 10.1007/s10554-019-01682-x, indexed in Pubmed:31414256.

12. Harris AW, Krieger EV, Kim M, et al. Cardiac Magnetic Resonance Imaging Versus Transthoracic Echocardiography for Prediction of Outcomes in Chronic Aortic or Mitral Regurgitation. Am J Cardiol. 2017; 119(7): 1074-1081, doi: 10.1016/j.amjcard.2016.12.017, indexed in Pubmed: 28153348.

13. Gabriel RS, Renapurkar R, Bolen MA, et al. Comparison of severity of aortic regurgitation by cardiovascular magnetic resonance versus transthoracic echocardiography. Am J Cardiol. 2011; 108(7): 1014-1020, doi: 10.1016/j. amjcard.2011.05.034, indexed in Pubmed: 21784393.

14. Kutty S, Whitehead KK, Natarajan S, et al. Qualitative echocardiographic assessment of aortic valve regurgitation with quantitative cardiac magnetic resonance: a comparative study. Pediatr Cardiol. 2009; 30(7):971-977, doi: 10.1007/s00246-009-9490-6, indexed in Pubmed: 19636486.

15. Dulce MC, Mostbeck GH, O'Sullivan M, et al. Severity of aortic regurgitation: interstudy reproducibility of measurements with velocity-encoded cine MR imaging. Radiology. 1992; 185(1): 235-240, doi: 10.1148/radiology.185.1.1523315, indexed in Pubmed: 1523315

16. Honda N, Machida $\mathrm{K}$, Hashimoto $\mathrm{M}$, et al. Aortic regurgitation: quantitation with MR imaging velocity mapping. Radiology. 1993; 186(1): 189-194, doi: 10.1148/radiology.186.1.8416562, indexed in Pubmed: 8416562

17. Søndergaard L, Hildebrandt $P$, Lindvig $K$, et al. Quantification of aortic regurgitation by magnetic resonance velocity mapping. Am Heart J. 1993; 125(4): 1081-1090, doi: 10.1016/0002-8703(93)90117-r, indexed in Pubmed: 8465731.

18. Bellenger NG, Burgess MI, Ray SG, et al. Comparison of left ventricular ejection fraction and volumes in heart failure by echocardiography, radionuclide ventriculography and cardiovascular magnetic resonance; are they interchangeable? Eur Heart J. 2000; 21(16): 1387-1396, doi: 10.1053/euhj.2000.2011, indexed in Pubmed: 10952828.

19. Hudsmith LE, Petersen SE, Francis JM, et al. Normal human left and right ventricular and left atrial dimensions using steady state free precession magnetic resonance imaging. J Cardiovasc Magn Reson. 2005; 7(5): 775-782, doi: 10.1080/10976640500295516, indexed in Pubmed: 16353438.

20. Uretsky S, Supariwala A, Nidadovolu $P$, et al. Quantification of left ventricular remodeling in response to isolated aortic or mitral regurgitation. J Cardiovasc Magn Reson. 2010; 12: 32, doi: 10.1186/1532-429X-12-32, indexed in Pubmed: 20497540.

21. Gocoł R, Malinowski M, Bis J, et al. Long-term outcomes of aortic valve repair in over 500 consecutive patients: a single-center experience. Kardiol Pol. 2020; 78(9): 861-868, doi: 10.33963/KP.15406, indexed in Pubmed: 32486628.

22. Łukaszewski M, Bielicki G, Berezowski $M$, et al. Aortic valve-sparing procedures in patients with aortic valve insufficiency: a singlecenter experience. Kardiol Pol. 2020; 78(2): 158-160, doi: 10.33963/KP.15152, indexed in Pubmed: 31969551 